\title{
Risk stratification for arrhythmic death in an emergency department cohort: a new method of nonlinear PD2i analysis of the ECG
}

\author{
James E Skinner ${ }^{1}$ \\ Michael Meyer ${ }^{2}$ \\ William C Dalsey ${ }^{3}$ \\ Brian A Nester ${ }^{4}$ \\ George Ramalanjaona ${ }^{5}$ \\ Brian J O'Neil ${ }^{6}$ \\ Antoinette Mangione ${ }^{7}$ \\ Carol Terregino ${ }^{8}$ \\ Abel Moreyra ${ }^{9}$ \\ Daniel N Weiss ${ }^{10}$ \\ Jerry M Anchin' \\ Una Geary" \\ Pamela Taggart ${ }^{12}$ \\ 'Vicor Technologies, Inc, Boca Raton, \\ FL, USA; ${ }^{2}$ Max Planck Institute for \\ Experimental Physiology, Goettingen, \\ Germany; ${ }^{3}$ Kimbal Medical Center, \\ Lakewood, NJ, USA; ${ }^{4}$ Lehigh Valley \\ Hospital and Health Network, \\ Allentown, PA, USA; ${ }^{5}$ St. Michaels \\ Hospital, Newark, NJ, USA; 'William \\ Beaumont Hospital, Royal Oak, MI, \\ USA; ${ }^{7}$ Luitpolb Pharmaceuticals, \\ Inc., Norristown, PA, USA; ${ }^{8}$ Cooper \\ Medical Center, Camden, NJ, USA; \\ 'Robert Wood Johnson Medical \\ School, New Brunswick, NJ, USA; \\ ${ }^{10}$ Florida Arrhythmia Consultants, \\ Ft. Lauderdale, FL, USA; "'St. James \\ Hospital, Dublin, Ireland; ${ }^{12}$ Albert \\ Einstein Medical Center, Philadelphia, \\ PA, USA
}

Correspondence: James E Skinner Vicor Technologies, Inc., 2300 Corporate Blvd, Ste I23, Boca Raton FL 33432, USA Tel + I 5708975797

Fax + I 56I 9952449

Email jskinner@vicortech.com

\begin{abstract}
Heart rate variability (HRV) reflects both cardiac autonomic function and risk of sudden arrhythmic death (AD). Indices of HRV based on linear stochastic models are independent risk factors for AD in postmyocardial infarction (MI) cohorts. Indices based on nonlinear deterministic models have a higher sensitivity and specificity for predicting AD in retrospective data. A new nonlinear deterministic model, the automated Point Correlation Dimension (PD2i), was prospectively evaluated for prediction of AD. Patients were enrolled $(\mathrm{N}=918)$ in 6 emergency departments (EDs) upon presentation with chest pain and being determined to be at risk of acute MI (AMI) $>7 \%$. Brief digital ECGs ( $>1000$ heartbeats, $\sim 15 \mathrm{~min}$ ) were recorded and automated PD2i results obtained. Out-of-hospital AD was determined by modified Hinkle-Thaler criteria. All-cause mortality at 1 year was $6.2 \%$, with $3.5 \%$ being ADs. Of the AD fatalities, 34\% were without previous history of MI or diagnosis of AMI. The PD2i prediction of $\mathrm{AD}$ had sensitivity $=96 \%$, specificity $=85 \%$, negative predictive value $=99 \%$, and relative risk $>24.2$ ( $\mathrm{p} \leq 0.001)$. HRV analysis by the time-dependent nonlinear PD2i algorithm can accurately predict risk of AD in an ED cohort and may have both life-saving and resource-saving implications for individual risk assessment.
\end{abstract}

Keywords: heart rate variability, sudden death, ventricular arrhythmias, chaos, non-linear

\section{Introduction}

Analysis of heart rate variability (HRV) noninvasively provides real-time trending of beat-to-beat changes in the ECG that reflect changes in cardiac autonomic function and performance (Askelrod et al 1981; Stein et al 1994). Abnormalities are indicated by low indices of HRV (Stein et al 1994). Such diminished measures are associated with increased susceptibility to ventricular arrhythmias and sudden arrhythmic death (AD) (Kleiger et al 1987; Stein et al 1994; Ho et al 1997).

Decreased HRV indices are frequently found among patients with myocardial infarction (acute MI [AMI] or post MI), congestive heart failure (CHF), diabetes, and alcoholic cardiomyopathy (Stein et al 1994). The indices are independent risk factors for mortality in the post MI and advanced CHF patients (Kleiger et al 1987; Coumel et al 1991; Stein et al 1994; Ho et al 1997). HRV indices based on brief observational windows (10-15 $\mathrm{min}$ ) have the same predictability for sudden AD as those based on 24-hour records (Fei et al 1996).

The various HRV indices have not been widely adopted because no single measurement has yet demonstrated sufficient statistical predictability of lethal arrhythmogenesis in individuals, ie, they have inadequate sensitivity and specificity to be of clinical utility. The limited performance is related to assumptions of the model underlying the algorithm used to measure the beat-to-beat variation. For example, an algorithm such as Standard Deviation of Normal to Normal beats (SDNN), which is 
based on a linear stochastic model, presumes the data variation is random and is distributed around a mean. We know, however, that heartbeat intervals have internal correlations, are not random, and thus violate the model assumptions. In contrast, an algorithm based on a nonlinear deterministic model presumes that the variation is caused physiologically and has no beat-to-beat errors. Thus, the nonlinear measure should be inherently more accurate because the data fit the underlying model.

The consideration about the fundamental nature of the cardiac rhythm and its measurement has stimulated the development of new HRV analyses (Wagner and Persson 1998), but has left the clinical utility an open issue. Comparative tests among algorithms in retrospective data suggest that the time-dependent nonlinear algorithms that treat the problem of nonstationary data show the greatest promise for clinical HRV analyses (Skinner et al 1998, 2007; Braun et al 1998; Marwan et al 2002). Our objective was to evaluate prospectively the ability of one such nonlinear method, the Point Correlation Dimension method (PD2i), to predict documented VF and presumed AD (Hinkle and Thaler 1982; Bigger et al 1999) in high-risk chest-pain patients upon presentation to the emergency department (ED).

\section{Methods}

\section{Study design}

The intended clinical data were from a random sample of consecutively enrolled patients at 6 urban teaching hospitals; for patient safety in a busy ED, not all subjects could be consecutive. Exclusions were patients with atrial fibrillation or pacemakers, as they do not have a normal sinus rhythm. Each subject was evaluated in the ED by a validated clinical protocol (Lee et al 1991) that incorporates history, clinical symptoms, and conventional ECG findings to determine risk for MI. Patients with risk $>7 \%$ for an AMI were enrolled after informed consent was obtained. A digital ECG was recorded by a small battery-powered device (Vicor PD2i Cardiac Analyzer, digitization 1,000 Hz/channel; band-pass 0.1-1000 Hz; noise 2 microvolts RMS). Each digital ECG (lead V3-V5) contained $>1,000$ heartbeats (15- to 20-min sample). All clinical evaluations were performed by an attending emergency physician who was blinded to the digital ECG data. Analyses of the high-resolution ECGs were made without knowledge of the clinical data.

Patient follow-ups for clinical events were performed at 3 months and 1 year. Death searches by the Social Security Administration (SSA) confirmed or complemented some in-patient and out-patient clinical data. Subgroups were assigned as in-hospital AMI or no AMI (no AMI) and history of prior MI (postMI) or no history of MI (no postMI).

The protocol was approved by each local Institutional Review Board and conformed to the principles outlined in the Declaration of Helsinki. The patient's identities were safeguarded within each hospital's database and only coded identifiers were used by the clinical investigators. A Data Safety and Monitoring Board of 5 independent ED physicians provided additional patient safety.

\section{Clinical outcome measures and classification}

Primary clinical outcome measures were: 1) all-cause death, 2) documented fatal arrhythmias, and 3) presumed arrhythmic deaths, using Hinkle-Thaler criteria (Hinkel and Thaler 1981) as more recently used by Bigger and associates (Bigger et al 1999) to assess mechanism of out-of-hospital deaths. A 3-member Events Committee blinded to the digital ECG data reviewed each death to ascertain its location, underlying cause, probable mechanism, and associated acute cardiac symptoms. When modified Hinkle-Thaler data were incomplete, available clinical information was used, including the in-hospital documentation of ventricular arrhythmias and abnormal ECGs and the occurrence of out-of-hospital death without evidence of respiratory obstruction, hemorrhage, infection, or stroke. The judged AD designation could be with or without documentation of defined arrhythmias (LaRovere et al 1998) or AMI (Beaglehole et al 1987).

\section{PD2i analyses (Vicor 2.0)}

For the automated PD2i analysis of the RR intervals, using Vicor 2.0 software (Vicor Technologies, Inc., 2300 Corporte Blvd, NW, Boca Raton, FL 33432-7345), the common rule developed by Swinney and associates (Kostelich and Swinney 1989) was used for determining the minimum number of data points $(\mathrm{N})$. Files containing too much noise for a valid nonlinear test were a priori excluded by examination with the \% $\mathrm{N}$ Test (Skinner et al 1991, 1993, 1994, 1998, 2000, 2007; Vybiral and Skinner 1993), which does not require removal of outliers, and the Randomizedphase Surrogate Test (Theiler 1988; Theiler et al 1992), which does (ie, arrhythmias and artifacts $>3$ SD were replaced by a linear spline). A priori exclusion is required for nonlinear algorithms, as they are sensitive to noise and it is known that randomized heartbeat intervals, such as those in patients with atrial fibrillation or high arrhythmia rates cannot be analyzed. Files passing these quantitative exclusionary tests were further examined and required by 
a Noise Consideration Algorithm (NCA) to have the background noise below the \pm 5 integer noise-tolerance level of PD2i. Such tolerated noise in nonstationary data with known degrees of freedom does not significantly alter the mean PD2i values (Skinner et al 1994).

The cut-point selected for the PD2i Test (minimum PD2 $\mathrm{i} \leq 1.4$ ) was based on our previous retrospective study in patients with nonsustained ventricular tachycardia (VT) (triplets to less than 30-sec runs of VT) in which the separator between the ventricular fibrillation (VF) and nonVF patients was clearly a minimum PD2i score in the 1.21 to $1.40 \mathrm{bin}$, that is, PD2i $\leq 1.4$ (Skinner et al Vybiral 1993). Receiver-Operator Curves (ROC) for the present data were used to determine the validity of the minimum PD2 $\mathrm{i} \leq 1.4$ cut-point.

\section{Statistical analysis}

Because the distribution of the outcomes could not be presumed to be normal, the nonparametric binomial-probability statistic or Fischer Exact Test for contingency tables was used to assess the significance of death-outcome prediction by the PD2i of the heartbeats. The sensitivity, specificity, negative predictive value, and relative-risk statistics assessed predictability, using Fisher's Exact Test to determine the significance of each true-false vs alive-deceased contingency. Power was determined using the proportions method of Fleiss (Fleiss 1981, power $>90 \%$ ).

Parametric t-tests were used to compare algorithmic results from RR data with their surrogates. Each test had $>1,000$ data points and an approximately unit-normal distribution (Schreiber and Schmitz 1996). One-tailed $\alpha$-levels were used for the surrogate tests, as the null hypothesis is directional.

The test for homogeneity between 2 enrollment periods was performed using the Breslow-Day Test for homogeneity. Other tests were used to express statistical significance of PD2i prediction of adjudicated AD: the Cochran-MantelHaenszel statistic (based on table scores) and estimates of common Relative Risk (Mantel-Haenszel statistics, and Logit, which is an odds ratio). For combining data from the two enrollment periods, a fixed-effects approach was used to sum across the probabilities for calculating contingency table statistics, using 3 different scenarios for counting ADs, including those ADs with rejected PD2i tests.

Cox proportional hazards models were fitted to determine what set of risk factors would predict AD. These models were fitted using a stepwise selection procedure in SAS Proc PHREG. All statistics were calculated by a professional statistician, using software packages of SAS and Prism.

\section{Results}

\section{Clinical characteristics}

The study enrolled a total of 918 patients, 335 between 1998 and 1999 and 583 between 2004 and 2006. Each of the 918 was an ED patient that had risk for AMI $>7 \%$ by the Lee et al (1991) chest-pain algorithm. There were 56 all-cause deaths within 1 year of follow-up, 35 in the 1998-1999 patients and 21 in the 2004-2006 patients. Of these 56 deaths $29(52 \%)$ were classified by the Events Committee as AD and 27 as nonAD.

Follow-up was relatively complete in the first enrollment period, as the SSA search of death certificates left only $8(2.3 \%)$ lost to follow-up. In the second enrollment period SSA assistance was not available and left $34(5.8 \%)$ lost to follow-up. These 42 patients were eliminated from study, the majority of which were PD2i-negative (36 PD2i-neg, 3 PD2i-pos, 3 PD2i-rej). Fifty-seven others either had no protocol ECGs recorded or withdrew consent, leaving 819 with complete data.

Table 1 shows the clinical characteristics of the combined study cohort and those parceled out because of loss of follow-up or no ECG or consent. Each row is either an average or percentage for the number in the column category. The two groups that could not be studied (columns 3 and 4) tended to be the same in their clinical characteristics as the population studied, except for a slightly higher percentage of males in the lost to follow-up (Chi-square, $\mathrm{p} \leq 0.01$ ).

\section{Contingency table for primary PD2i results}

Table 2 shows achievement of high sensitivity, specificity, negative predictive value, and relative risk statistics for the

Table I Clinical characteristics of study cohort

\begin{tabular}{|c|c|c|c|}
\hline $\begin{array}{l}\text { Clinical } \\
\text { characteristic }\end{array}$ & $\begin{array}{l}\text { Enrolled } \\
\text { patients } \\
\mathbf{N}=918\end{array}$ & $\begin{array}{l}\text { Lost to } \\
\text { follow-up } 2 \\
N=42\end{array}$ & $\begin{array}{l}\text { No ECG } \\
\text { or consent }{ }^{3} \\
\mathbf{N}=\mathbf{5 7}\end{array}$ \\
\hline Average age & 63 & 51 & 66 \\
\hline Average LVEF & 50 & 52 & 47 \\
\hline$\%$ Male & $52 *$ & $75^{*}$ & 58 \\
\hline \% Diabetic & 23 & 31 & 25 \\
\hline \% Hypertension & 57 & 53 & 55 \\
\hline$\% \mathrm{CHF}$ & 10 & 7 & 11 \\
\hline \% Prior MI & 25 & 21 & 28 \\
\hline$\%$ Current AMI & 21 & 14 & 26 \\
\hline
\end{tabular}

'Caucasian 73\%,Afro-American 24\%, [Hispanic (all nonAfro) 7\%], Asian 3\%. ${ }^{2}$ Lost to follow-up (4.6\%); 36 PD2i-Neg; 3 PD2i-Pos, 3 PD2i-Rej.

${ }^{3} \mathrm{No}$ ECG recorded or patient withdrew consent $(6.2 \%)$.

*Different from enrolled, $\mathrm{p} \leq 0.00 \mathrm{I}$ (Chi-square); all others are nonsignificant.

Abbreviations: $\mathrm{AMI}$, acute $\mathrm{Ml}$; $\mathrm{CHF}$, congestive heart failure; $\mathrm{MI}$, myocardial infarction; LVEF, left ventricular ejection fraction. 
Table 2 Contingency table for primary results

\begin{tabular}{|c|c|c|}
\hline & $\begin{array}{l}6 \text { Tertia } \\
\text { al enrol }\end{array}$ & $\begin{array}{l}\text { dy } \\
\text { hospitals } \\
\text { completed study }\end{array}$ \\
\hline & AD & no AD \\
\hline PD2i Pos & $26 \mathrm{TP}$ & $103 \mathrm{FP}$ \\
\hline PD2i Neg & I FN & $595 \mathrm{TN}$ \\
\hline Sensitivity $=26 /$ & ) $=96 \%$ & \\
\hline Specificity $=595$ & $+103)=$ & \\
\hline Negative predict & $\mathrm{ue}=595$ & I) $=99 \% *$ \\
\hline Positive predicti & $e=26 /($ & 3) $=20 \%$ \\
\hline Relative risk $=2$ & $(595+I)$ & $03)=24.2^{*}$ \\
\hline
\end{tabular}

$*_{\mathrm{p}}<0.01$, Fisher's Exact Test for contingency tables.

Abbreviations: $A D$, arrhythmic death; TP, true positive; FP, false positive; FN, false negative; TN, true negative.

combined enrollment periods. These results are dependent, however, upon the considerations of noise in the RR data. The 89 PD2i-rejected patients, of the 819 patients with complete data (10.86\%), were rejected by both the Randomized Phase Surrogate Test (inverse Fourier with random phases) and the $\% \mathrm{~N}$ Test (\% accepted PD2i/total PD2i). The \%N Test does not require the removal of outliers in the $\mathrm{RR}$ interval data, as does the Surrogate Test. Both tests excluded the same subjects. These PD2i-rejected subjects included all cases of supraventricular arrhythmia and/or ventricular ectopy, with net arrhythmia rates being $>10 \%$ of beats.

The sensitivity at 30 days was $100 \%$, specificity $80 \%$, and relative-risk $>22.4$ ( $\mathrm{p} \leq 0.001$, power $>90 \%)$; these values are similar to those at 365 days: sensitivity $=96 \%$, specificity $=85 \%$; relative-risk $>24.2(\mathrm{p} \leq 0.001$, power $>90 \%)$. Relative-risk is a statistic that considers the true-positive/ false-negative ratio (TP/FN) adjusted for test proportions, and it emphasizes the impact of the medically undesirable FN type of error. The single FN is a result that also gives the negative predictive value such a high percentage (99\%). The positive predictive value was not significant, as only 26 of the 129 PD2i-positive cases died. The 103 false-positives (FPs), however, were in hospital and treated (angina, 3; unstable angina, 23; coronary artery disease, 38; AMI, 11; cardiac heart failure, 22).

PD2i was also found to be a statistically significant predictor of $\mathrm{AD}$ in the $\mathrm{AMI}$ subgroup (relative-risk = 7.39, $\mathrm{p} \leq 0.001$ ), but not in the postMI subgroup. Beta-blocker therapy did not completely prevent $\mathrm{AD}$, but tended to reduced its rate: 6 ADs in the 384 patients on beta-blockers; 23 ADs in the 435 patients not on beta-blockers.

The Cox proportional hazards model examined risk factors. The following variables were considered: age, gender, current beta-blocker use, concomitant beta-blocker use, diagnosis of AMI, cardiac heart failure, unstable angina, angina, or history of coronary artery bypass graph, catheterization, coronary artery disease, diabetes mellitus, hypertension, previous MI, and the PD2i Test. Two models were fitted to the data using separate approaches, one including the enrollment period as a covariate forced into the model, and one where enrollment period was considered as another possible covariate, but not forced into the model. The results indicated that enrollment period was not predictive of AD; the model where it was included was very similar to the one where it was not included. In each model there were only 3 variables that were identified as significant predictors of AD - age, diagnosis of AMI, and PD2 $\mathrm{i}$ Test. In the model with enrollment period included as a covariate, the hazard ratio for PD2i Test was 95.6 $(\mathrm{p} \leq 0.0001)$, for age was $1.04(\mathrm{p} \leq 0.01)$ and for AMI was $5.0(\mathrm{p} \leq 0.0001)$.

Table 3 shows the distribution of the outcomes (columns) expressed according to the clinical characteristics (rows) for the patients with complete data. There were 89 who had PD2i Test rejection due to high arrhythmia rate (11\%), and these contained 2 with AD. The percentages in column 2 are distributed for each row in the columns to the right. For example, $21 \%$ of the patient population had documented AMI, and the majority $(18 \%)$ were PD2i-negative and did not manifest AD. The $100 \%$ of the PD2i tests had $11 \%$ rejected, with the remainder distributed across the outcome groups.

\section{Test for homogeneity of the two enrollment groups}

The test for homogeneity is presented for each of three ways to consider the impact of the two AD patients with their PD2 $\mathrm{i}$ tests rejected:

1) Standard Analysis (S) uses only data where the PD2i device gave a positive or negative reading (ADs with rejected PD2i Test are not considered);

2) Conservative Analysis (C) uses the data from the first analysis, but considers the two ADs as PD2i failures;

3) Best Analysis (B) uses the data from the first analysis, but considers the two ADs as PD2i successes.

The third approach would give the highest (best) possible predictive results for the PD2i device, but it is biased toward statistical significance and included only for comparison with the other two. The Breslow-Day Test results for the three scenarios are shown in Table 4. There were no significant probabilities to suggest that the two enrollment periods are different, therefore the two enrollment groups may be combined. 
Table 3 Clinical characteristics and outcome distributions

\begin{tabular}{|c|c|c|c|c|c|}
\hline \multirow{2}{*}{$\begin{array}{l}\text { Clinical } \\
\text { characteristic }\end{array}$} & \multirow{2}{*}{$\begin{array}{l}\text { All } \\
\text { patients' }\end{array}$} & \multicolumn{2}{|c|}{$\begin{array}{l}\text { PD2i } \\
\text { positive }\end{array}$} & \multicolumn{2}{|c|}{$\begin{array}{l}\text { PD2i } \\
\text { negative }\end{array}$} \\
\hline & & Alive $^{2}$ & Died $^{3}$ & Alive $^{4}$ & Died $^{5}$ \\
\hline \% PD2i Test** & 100 & 13 & 3 & 73 & 0.1 \\
\hline Average age* & 63 & 62 & 72 & 61 & 73 \\
\hline Average LVEF & 50 & 52 & 34 & 50 & None \\
\hline$\%$ Male & 52 & 6 & 2 & 44 & 0 \\
\hline \% Diabetic & 23 & 4 & I & 18 & 0 \\
\hline \% Hypertension & 57 & 9 & 2 & 46 & 0 \\
\hline$\% \mathrm{CHF}$ & 10 & 3 & 1 & 6 & 0 \\
\hline \% Prior MI & 25 & 3 & I & 21 & 0 \\
\hline$\%$ Current AMI** & 21 & 1 & 2 & 18 & 0.1 \\
\hline
\end{tabular}

'819 with complete data; $11 \%$ had PD2i Test rejection (2ADs).

${ }^{2} 103$ False positives

${ }^{3} 26$ True positives

4595 True negatives

${ }^{5}$ I False negative

*p $\leq 0.01, * * p \leq 0.001$ (Cox Proportional Hazards Model)

Abbreviations: $\mathrm{AMI}$, acute $\mathrm{Ml}$; $\mathrm{CHF}$, congestive heart failure; $\mathrm{MI}$, myocardial infarction; LVEF, left ventricular ejection fraction.

The overall odds ratio is very large and highly significant for each of the three scenarios, suggesting that indeed there is a strong relationship between the PD2i positive results and mortality and the PD2i negative results and patients surviving (Cochran-Mantel-Haenszel: S, 52.8947; C, 26.8287; B, 78.9448; $\mathrm{p} \leq 0.0001)$. Also the estimates of the common relative risk for each scenario are highly significant (case control: S, 57.5742; C, 15.0840; B, $79.6052 ; \mathrm{p} \leq 0.001)$.

\section{Receiver-operator curve for examination of PD2i cut-point}

Figure 1 shows the ROC plotted for all data with positive or negative PD2i tests. The ROC examines various cut-points for the PD2i Test to find the one that maximizes sensitivity and specificity. There is a sharp break in the ROC at the cut-point, PD2i $\leq 1.4$. The sensitivity (96\%) and specificity $(85 \%)$ are maximized at this value, with the area under the curve being large (area $=0.9225)$.

\section{Sample RR intervals and PD2i}

Figure 2 shows sample data from two typical ED patients. One suffered AD within 24 hours and the other was discharged from the hospital after 24 hours with a diagnosis of esophageal reflux, so is a normal control (CONTROL). Note in the PD2i trace of the AD patient that a relatively sustained excursion occurs (underline), that is followed by a PD2i increase and then a transient low-dimensional excursion (arrow). These two PD2i patterns each indicate PD2i $\leq 1.4$ and are typical of what constitutes a positive PD2i test.

\section{All-cause death}

Figure 3 shows the accumulative all-cause deaths as a function of the time of death after the ECG recording. Most ADs occurred within 180 days after the recording of the ECG. Most of the nonAD deaths (unfilled squares) occurred in elderly subjects (Elderly, mean age 77 years) and after 180 days. Those nonAD deaths that occurred before 180 days had other common mechanisms of death. Eight of the ADs at admission were referred to low-level hospitalization (Telemetry).

\section{Composite RR interval and PD2i results}

Figure 4 shows the composite RR data and corresponding PD2i values for the first $18 \mathrm{AD}$ patients that had both the ECG and follow-up completed and were not rejected by Surrogate or $\% \mathrm{~N}$ Tests (the single False Negative case is omitted, as 2 of 3 clinical ECGs around the time of the ECG recording were normal). These $\mathrm{AD}$ patients are compared with $18 \mathrm{AMI}$ subjects, associated in both time and ED site with the first 18 , but none of whom manifested AD within the 1 year of follow-up. More than $95 \%$ of the accepted PD2is were above 3.0 for the AMI controls, whereas $95 \%$ were below this value for the AD patients (Binomial Probability Test, $\mathrm{p}<0.001$; sensitivity $=100 \%$; specificity $=100 \%$; power $>90 \%$ ).

\section{Discussion}

\section{Interpretation of results}

The present prospective results confirm our earlier studies in retrospective data that compared PD2i, Approximate Entropy, Detrended Fluctuation Analysis, and other nonlinear HRV algorithms for risk stratification (Skinner et al 1993, 1998; Vybiral and Skinner 1993). Similar comparison of PD2i with other competing algorithms, including SD of

Table 4 Test for homogeneity of enrollment groups

Breslow-Day Test for homogeneity of the odds ratios using three scenarios $^{a}$

\begin{tabular}{ll}
\hline Chi-square & S 0.926 I \\
& C 2.5088 \\
DF & B I.4552 \\
& S I \\
& C I \\
Pr $>$ Chi-square & B I \\
& S $0.3359^{b}$ \\
& C $0.1132^{b}$ \\
& B $0.2277^{b}$ \\
\hline
\end{tabular}

${ }^{a} \mathrm{~S}=$ Standard $; \mathrm{C}=$ Conservative B $=$ Best.

${ }^{b}$ not statistically significant. 


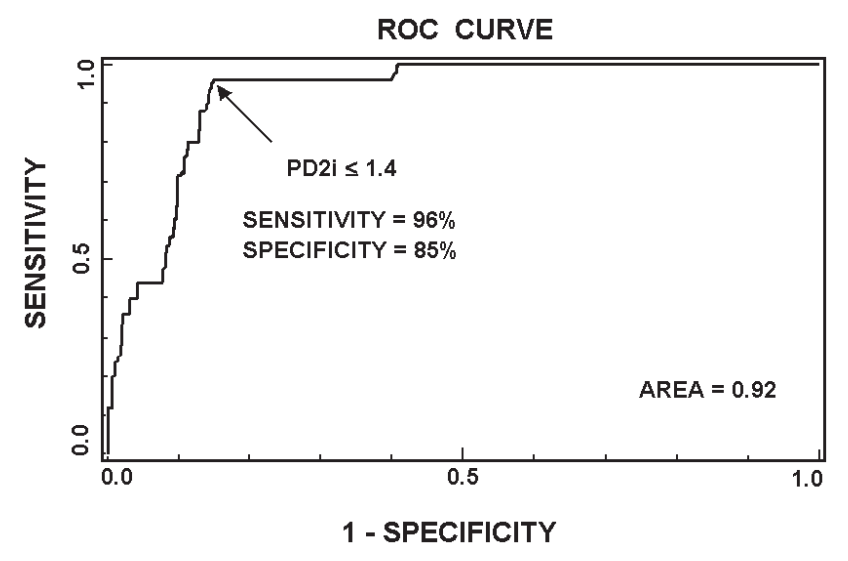

Figure I Receiver-operator curve (ROC) for all data with a positive or negative PD2i Test. The ROC determines the criterion cut-point for the PD2i Test that maximizes the sensitivity and specificity. At a cut-point of PD2i $\leq 1.4$, the maximum sensitivity and specificity are found with the area under the ROC being high (0.92).

normal beats and power spectral ratios, was made for the first enrollment period subjects and published separately (Skinner 2007). In both the retrospective and prospective studies, the PD2i algorithm had the superior sensitivity and specificity in all subgroups.

Findings in public data, in which the ECGs were downloaded from the internet (PhysioBank; 22 VT/VF patients and 22 arrhythmia controls), also showed similarly high sensitivity and specificity for the PD2i Test (Skinner et al 2008).

The $\% \mathrm{~N}$ Test was used in all of the previous studies to reject data unsuitable for nonlinear analysis because of noise content, including the type of noise that results from randomized RR intervals. The $\% \mathrm{~N}$ Test in this study rejected the same subjects as those rejected by the more complicated Surrogate Test. The $\% \mathrm{~N}$ Test does not require removal of arrhythmias, artifacts, and other nonstationarities from the data. So it would appear to be a sufficient test with fewer steps in the calculation.

The ROC confirms the PD2 $\mathrm{i} \leq 1.4$ cut-point used in this and all previous studies. The previous retrospective studies all predicted VF/VT within 24 hours. The prospective studies, including the present one, show that PD2i prediction of AD extends outward from days to weeks and months.

\section{Limitations}

Nonlinear algorithms require that there be little or no noise in the data. Determining the exact methodology for handling noise in PD2i analysis of heartbeats remains a challenge. Consideration of low-level noise is necessary for achieving the high sensitivity and specificity of PD2i prediction of AD. Even better methods may eventually be found. Patients with atrial fibrillation and arrhythmia rates greater than $10 \%$ of beats are currently excluded, as they should be, because of the randomization that occurs in their heartbeat intervals.

\section{What the PD2i measures}

There can be little doubt that cardiac vulnerability to lethal arrhythmogenesis is regulated by the brain and autonomic nervous system. In animals, blockade of the descending efferent pathways at the level of: 1) the frontal cortex (Skinner and Reed 1981) or amygdala (Carpeggiani et al 1991), 2) the peripheral cardiac nerves (Ebert et al 1970), or 3) locations between these two sites, in the hypothalamus, mesencephalon, or medulla (Skinner and Reed 1981), will each prevent VF after left anterior descending coronary artery occlusion. These same higher neural systems also participate in the regulation of heart rate: 1) the afferent-efferent loops through the medulla underlie the baroreflex and respiratory sinus arrhythmia; 2) the loops through the hypothalamus regulate the affects of blood $\mathrm{pH}$ and temperature; and 3) the loops through the frontal cortex and amygdala regulate cerebral defensive reactions (Skinner 1985).

During quiet wakefulness each of these multiple loops contributes to the regulation of the RR intervals by its
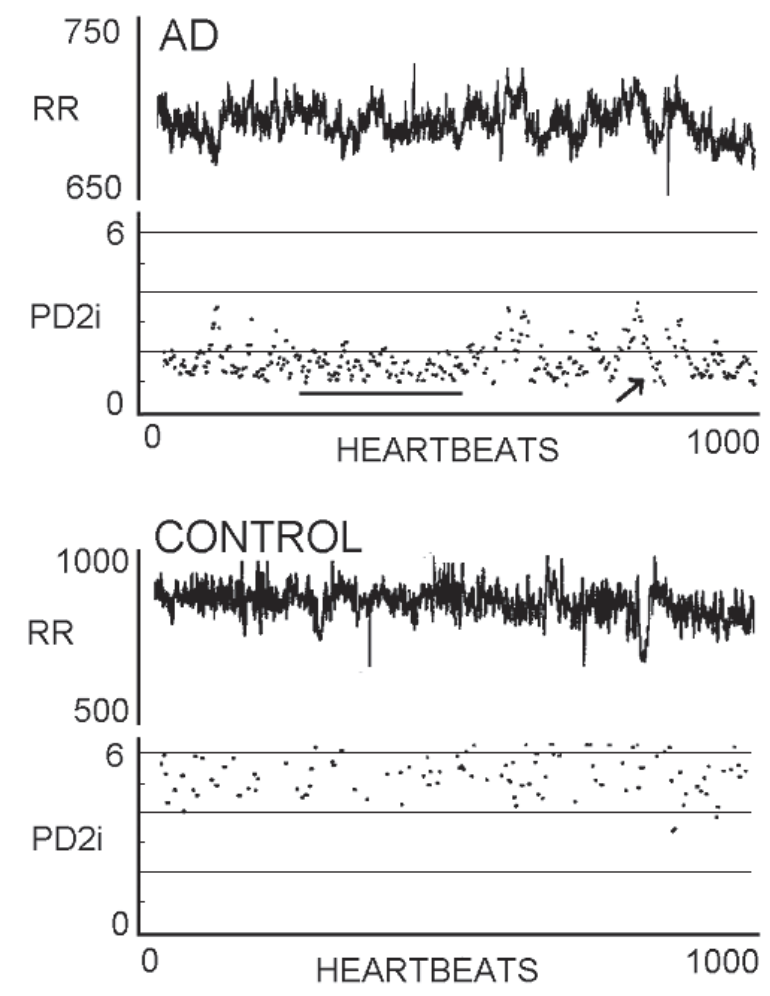

Figure 2 RR intervals and associated PD2is from two types of patients. Upper panel shows RR data and corresponding PD2i results from a patient who experienced documented arrhythmic death (AD) within 24 hours; note the two patterns of either a sustained low-dimension (line) or the systematic low-dimensional excursion (arrow). The lower panel shows data and results from a normal patient discharged after diagnosis of gastro-esophageal reflux disorder (CONTROL). 


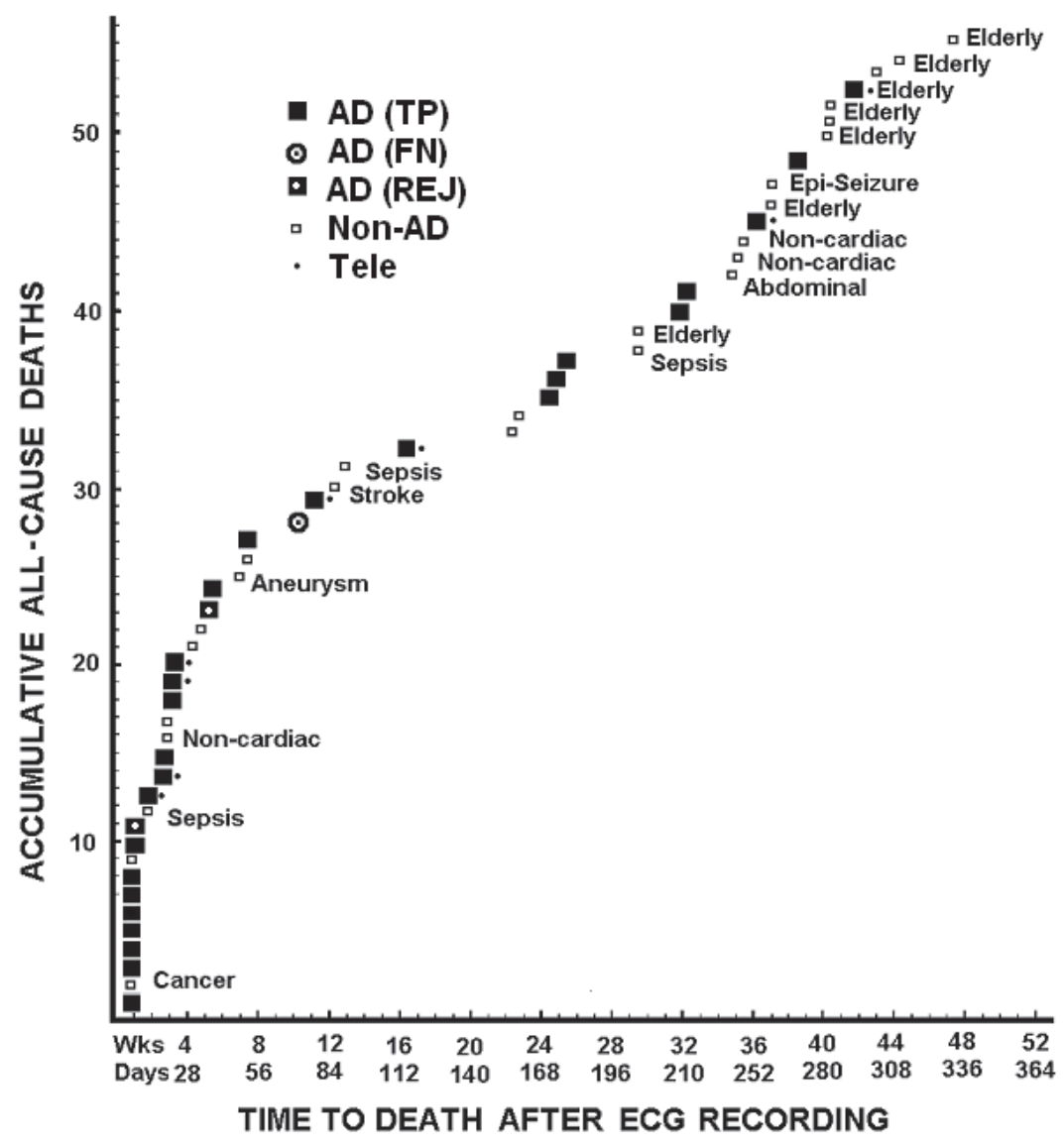

Figure 3 All-cause death vs time of occurrence after the brief ECG recording. Large squares indicate cardiac arrhythmic death (AD), as adjudicated by the Events Committee. All non-AD deaths are indicated by small unfilled squares, and include deaths due to cancer, sepsis, stroke, etc. AD patients admitted to low-level telemetry observation at admission are indicated by dots.

interaction on its own time scale. The degrees of freedom resulting from the net interactions are approximately 5-6 in the human (Figure 2, CONTROL). This type of tiedtogether regulation results in the erratic RR-interval series that is clinically referred to as the "normal sinus rhythm". It is the physiological evolution of abnormal dynamics in this tied-together regulation that leads to the type of altered HRV that is associated with susceptibility to AD in hospitalized patients. This evolved dynamic is associated with a reduction in the number of degrees of freedom (dimensions), as shown in Figures 2-4. The reduction in the degrees of freedom in the heartbeats could result from greater "cooperation" or phase relationship among the independent regulators, each of which accounts for one degree of freedom. We do not yet know how this or any alternative mechanism may actually produce the changed dynamics.

The PD2 $\mathrm{i}$ algorithm is a new measure of heartbeat regulation that, instead of being based on a linear stochastic model like the mean, standard deviation, or power spectra, is a nonlinear measure of variability not requiring any a priori presumptions about an underlying model. The motivation for developing the PD2i algorithm was the simple observation that the series of heartbeat intervals is not a sequence of independent numbers randomly distributed around a mean, as is required by any algorithm based on a linear stochastic model. An in-depth discussion of these fundamental issues ("When the mean is meaningless") is provided elsewhere (Steidl and Meyer 2002, 2003a, b). The poorer performance of the linear measures, we believe, results from the fundamental misconception that analyzing an inherently nonlinear and correlated time series by a linear technique is all right. On theoretical grounds this usage commits a serious type-II error.

The heart transplant recipient manifests a resting heartbeat dynamics of PD2i = 1.0, with little beat-to-beat variation, although it can slowly change with exercise (Meyer et al 1996). This denervation result indicates that the nervous system produces the higher degrees of freedom found in the heartbeat intervals of the intact subject. Besides the 

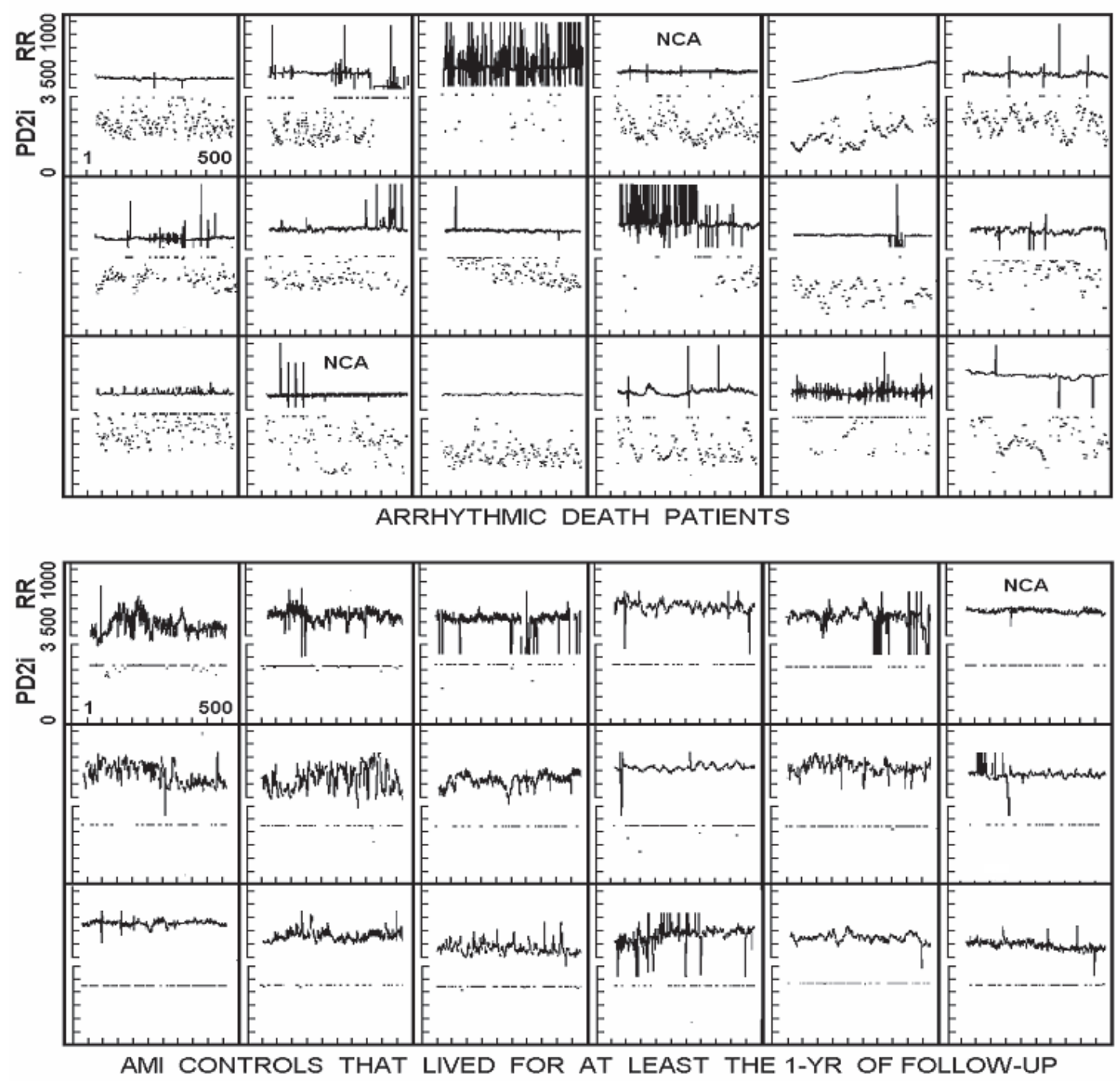

Figure 4 Composite RR and PD2i results for the first 18 arrhythmic death (AD) cases with all clinical data and no a priori exclusions for noise contamination. NCA = cases in which a single bit (noise-bit) was removed from each data point thus forcing the background noise level below \pm 5 integers. In the acute myocardial infarction (AMI) controls all PD2i $>3.0$ are pushed down to the 3.0 line for display.

denervation, the lowering of the heartbeat PD2i to 1.0 can be achieved in animals by blockade of the N-methyl-D-aspartate (NMDA) receptors, which are the synaptic mediators in virtually all of the higher cognitive and autonomic regulatory centers in the brain (Skinner et al 2000).

In summary, the PD2i measures the time-dependent degree of interaction or "cooperation" among the competing neural loops that simultaneously control the dynamics of the cardiac rhythm and the vulnerability to lethal arrhythmogenesis. Breakdown of the independent coupling among these competing autonomic nervous loops (ie, PD2i $\leq 1.4$ ) sets the stage for the evolution of lethal arrhythmogenesis. The mechanism for the coordination of such "cooperation," although unknown, is certainly neural, as there can be little doubt that the coordination is organized within the brain and is projected onto the nodal and ventricular tissues of the heart through the autonomic nervous system.

\section{Conclusions}

We conclude that a rapid, accurate, and cost-effective assessment of risk of sudden AD can be made by the PD2i Test (Vicor 2.0) for patients presenting in the Emergency Department with a risk of AMI $>7 \%$. The $>99 \%$ negative predictive value for AD indicates that the PD2i Test is able to define a very low risk population.

\section{Acknowledgments}

This work was supported in part by grants from the National Institutes of Health, NS 27745 and HL 069563. 


\section{Disclosures}

JE Skinner, DL Weiss, and JM Anchin are employees of Vicor Technologies, Inc., and are company shareholders.

\section{References}

Akselrod S, Gordon D, Ubel FA, et al. 1981. Power spectrum analysis of heart rate fluctuation: a quantitative probe of beat to beat cardiovascular control. Science, 213:220-2.

Beaglehole R, Stewart AW, Butler M. 1987. Comparability of old and new World Health Organization criteria for definite myocardial infarction. Intl J Epidemiol, 16:373-6.

Bigger JT Jr, Whang W, Rottman JN, et al. 1999. Mechanisms of death in the CABG Patch trial: a randomized trial of implantable cardiac defibrillator prophylaxis in patients at high risk of death after coronary artery bypass graft surgery. Circulation, 99:1416-21.

Braun C, Kowallik P, Freking A, et al. 1998. Demonstration of nonlinear components in heart rate variability of healthy persons. Am J Physiol, 275:H1577-84

Carpeggiani C, Landisman C, Montaron M-F, et al. 1992. Cryoblockade in limbic brain (amygdala) delays or prevents ventricular fibrillation following coronary artery occlusion in psychologically stressed pigs. Circ Res, 70:600-6.

Coumel PH, Hermida JS, Wennerblöm B, et al. 1991. Heart rate variability in myocardial hypertrophy and heart failure, and the effects of betablocking therapy: a non-spectral analysis of heart rate oscillations. Eur Heart J, 12:412-22.

Ebert PA, Vanderbeck RB, Allgood RJ, et al. 1970. Effect of chronic cardiac denervation on arrhythmias after coronary artery ligation. Cardiovasc Res, 4:141-7.

Elbert T, Ray WJ, Kowalik ZJ, et al. 1994. Chaos and Physiology. Physiol Rev, 74:1-47.

Fei L, Copie X, Malik M, et al. 1996. Short- and long-term assessment of heart rate variability for risk stratification after acute myocardial infarction. Am J Cardiol, 77:681-4.

Fleiss JL. 1981. Statistical Methods for Rates and Proportions. Second Edition, John Wiley and Sons, New York. Table A.3. Sample sizes per group for a two-tailed test on proportions. pp. 260-80.

Hinkle LE Jr, Thaler HT. 1982. Clinical classification of cardiac deaths. Circulation, 65:457-64.

Ho KK, Moody GB, Peng CK, et al. 1997. Predicting survival in heart failure case and control subjects by use of fully automated methods for deriving nonlinear and conventional indices of heart rate dynamics. Circulation, 96:842-8.

Kleiger RE, Miller JP, Bigger JT, et al: The Multicenter Post- Research Group. 1987. Decreased heart rate variability and its association with increased mortality after acute myocardial infarction. Am J Cardiol, 59:256-62.

Kostelich EJ, Swinney HL. 1989. Practical considerations in estimating dimension from time series data. Physica Scripta, 40:436-41.

La Rovere MT, Bigger JT Jr, Marcus FI, et al; Autonomic Tone and Reflexes After Myocardial Infarction Investigators. 1998. Baroreflex sensitivity and heart rate variability in prediction of total cardiac mortality after myocardial infarction. Lancet, 351:478-84.

Lee TH, Juarez G, Cook EF, et al. 1991. Ruling out myocardial infarction: a prospective multicenter validation of a 12-hour strategy for patients at low risk. New Engl J Med, 328:1239-46.

Marwan N, Wessel N, Meyerfeldt U, et al. 2002. Recurrence-plot-based measures of complexity and their application to heart-rate-variability data. Phys Rev E, 66:02670-2.
Meyer M, Marconi C, Ferretti, et al. 1996. Heart rate variability in the human transplanted heart: nonlinear dynamics and QT vs RR-QT alterations during exercise suggest a return of neurocardiac regulation in long-term recovery. Integ Physiol Behav Sci, 31:289-305.

Schreiber T, Schmitz A. 1996. Improved Surrogate Data for Nonlinearity Tests. Phys Rev Lett, 77:635-8.

Skinner JE, Reed JC. 1981. Blockade of a frontocortical- brainstem pathway prevents ventricular fibrillation of the ischemic heart in pigs. $\mathrm{Am} \mathrm{J}$ Physiol, 240:H156-H16.

Skinner JE, 1985. The regulation of cardiac vulnerability by the cerebral defense system. J Amer Coll Cardiol, 5:88B-94B.

Skinner JE, Carpeggiani C, Landisman CE, et al. 1991. The correlation dimension of heartbeat intervals is reduced in conscious pigs by myocardial ischemia. Circulation Res, 68:966-76.

Skinner JE, Francis M, Pratt CM. 1993. Conventional heart rate variability analysis of ambulatory electrocardiographic recordings fails to predict imminent ventricular fibrillation. J Amer Coll Cardiol, 322:557-65.

Skinner JE, Pratt CM, Vybiral T. 1993. A reduction in the correlation dimension of heartbeat intervals proceeds imminent ventricular fibrillation in human subjects. Amer Heart J, 125:731-43.

Skinner JE, Molnar M, Tomberg C. 1994. The point correlation dimension: performance with nonstationary surrogate data and noise. Integr Physiol Behav Sci, 29:217-34.

Skinner JE, Zebrowski JJ, Kowalik ZJ. 1998. New nonlinear algorithms for analysis of heart rate variability: low dimensional chaos predicts lethal arrhythmias. In: Kantz H, Kurths J, Mayer-Kress G (Eds.) Nonlinear Analysis of Physiological Data. Springer, New York. pp. 129-66.

Skinner JE, Molnar M. 1999. Event-related dimensional reduction in the primary auditory cortex of the conscious cat are revealed by new techniques for enhancing the non-linear dimensional algorithms. Intern $J$ Psychophysiol, 34:21-35.

Skinner JE, Nester BA, Dalsey WC. 2000. Nonlinear dynamics of heart rate variability during experimental hemorrhage in ketamine-anesthetized rats. Amer J Physiol, 297:H1669-H78.

Skinner JE. 2007. New paradigms in heart-brain medicine: nonlinear physiology and state-dependent proteomics. Clev Clinc J Med, 74: S79-S85.

Skinner JE, Anchin JM, Weiss DN. 2008. Nonlinear analysis of the heartbeats in public patient ECGs using an automated PD2i algorithm for risk stratification of arrhythmic death. Therap Clin Risk Manag, In press.

Stein PK, Bosner MS, Kleiger RE, et al. 1994. Heart rate variability: a measure of cardiac autonomic tone. Am Heart J, 127:1376-80.

Stiedl O, Meyer M. 2002. Fractal dynamics of heart beat interval fluctuations in corticotropin-releasing factor receptor subtype 2 deficient mice. Int Physiol Behav Sci, 37:311-45.

Stiedl O, Meyer M. 2003a. Cardiac dynamics in corticotropin-releasing factor receptor subtype-2 deficient mice. Neuropeptides, 37:3-16.

Stiedl O, Meyer M. 2003b. Fractal dynamics in circadian cardiac time series of corticotropin-releasing factor receptor subtype-2 deficient mice. J Math Biol (online: 19 March 2003)

Theiler JT. 1998. Quantifying chaos: practical estimation of the correlation dimension. PhD Thesis, California Institute of Technology. Pasadena, California.

Theiler J, Eubank S, Longtin A, et al. 1992. Testing for nonlinearity in time series: the method of surrogate data. Physica D, 58:77-94.

Vybiral T, Skinner JE. 1993. The point correlation dimension of R-R intervals predicts sudden cardiac death among high-risk patients. Comput Cardiol, 257-60.

Wagner CD, Persson PB. 1998. Chaos in the cardiovascular system: an update. Cardiovasc Res, 40:257-64. 
